\title{
A New Approach to Universality Limits at the Edge of the Spectrum
}

\author{
D. S. Lubinsky \\ Dedicated to the 60th birthday of Percy Deift
}

\begin{abstract}
We show how localization and smoothing techniques can be used to establish universality at the edge of the spectrum for a fixed positive measure $\mu$ on $[-1,1]$. Assume that $\mu$ is a regular measure, and is absolutely continuous in some closed neighborhood $J$ of 1 . Assume that in $J, \mu^{\prime}(x)=$ $h(x)(1-x)^{\alpha}(1+x)^{\beta}$, where $h(1)>0$ and $h$ is continuous at 1 . Then universality at 1 for $\mu$ follows from universality at 1 for the classical Jacobi weight $(1-x)^{\alpha}(1+x)^{\beta}$.
\end{abstract}

\section{Results}

Let $\mu$ be a finite positive Borel measure on $(-1,1)$. Then we may define orthonormal polynomials

$$
p_{n}(x)=\gamma_{n} x^{n}+\ldots, \quad \gamma_{n}>0,
$$

$n=0,1,2, \ldots$ satisfying the orthonormality conditions

$$
\int_{-1}^{1} p_{n} p_{m} d \mu=\delta_{m n}
$$

These orthonormal polynomials satisfy a recurrence relation of the form

$$
x p_{n}(x)=a_{n+1} p_{n+1}(x)+b_{n} p_{n}(x)+a_{n} p_{n-1}(x),
$$

where

$$
a_{n}=\frac{\gamma_{n-1}}{\gamma_{n}}>0 \text { and } b_{n} \in \mathbb{R}, \quad n \geq 1
$$

and we use the convention $p_{-1}=0$. Throughout $w=\frac{d \mu}{d x}$ denotes the absolutely continuous part of $\mu$. A classic result of E. A. Rakhmanov [6] asserts that if $w>0$ a.e. in $[-1,1]$, then $\mu$ belongs to the Nevai-Blumenthal class $\mathcal{M}$, that is

$$
\lim _{n \rightarrow \infty} a_{n}=\frac{1}{2} \text { and } \lim _{n \rightarrow \infty} b_{n}=0 \text {. }
$$

1991 Mathematics Subject Classification. 15A52, 42 C05.

Research supported by NSF grant DMS0400446 and US-Israel BSF grant 2004353.

(C)0000 (copyright holder) 
A class of measures that contains $\mathcal{M}$ is the class of regular measures [7], defined by the condition

$$
\lim _{n \rightarrow \infty} \gamma_{n}^{1 / n}=2 .
$$

One of the key limits in random matrix theory, the so-called universality limit [1], involves the reproducing kernel

$$
K_{n}(x, y)=\sum_{k=0}^{n-1} p_{k}(x) p_{k}(y)
$$

and its normalized cousin

$$
\widetilde{K}_{n}(x, y)=w(x)^{1 / 2} w(y)^{1 / 2} K_{n}(x, y) .
$$

In $[\mathbf{3}]$, we presented a new approach to this universality limit, proving:

TheOREM 1.1. Let $\mu$ be a finite positive Borel measure on $(-1,1)$ that is regular. Let I be a closed subinterval of $(-1,1)$ such that $\mu$ is absolutely continuous in an open interval containing $I$.

(a) Assume that $w$ is positive and continuous at each point of I. Then

$$
\lim _{n \rightarrow \infty} \frac{\widetilde{K}_{n}\left(x+\frac{a}{\widetilde{K}_{n}(x, x)}, x+\frac{b}{\widetilde{K}_{n}(x, x)}\right)}{\widetilde{K}_{n}(x, x)}=\frac{\sin \pi(a-b)}{\pi(a-b)},
$$

uniformly for $x \in I$ and $a, b$ in compact subsets of the real line.

(b) Assume that $w$ is bounded above and below by positive constants, and moreover,

$w$ is Riemann integrable in $I$. Then if $p>0$ and $I^{\prime}$ is a closed subinterval of $I^{0}$,

$$
\lim _{n \rightarrow \infty} \int_{I^{\prime}}\left|\frac{\widetilde{K}_{n}\left(x+\frac{a}{\widetilde{K}_{n}(x, x)}, x+\frac{b}{\widetilde{K}_{n}(x, x)}\right)}{\widetilde{K}_{n}(x, x)}-\frac{\sin \pi(a-b)}{\pi(a-b)}\right|^{p} d x=0,
$$

uniformly for $a, b$ in compact subsets of the real line.

We also established $L_{1}$ analogues assuming less on $w$. In this paper, we show how localization and smoothing can be applied at the edge 1 of the spectrum. As far as the author is aware, the most general result to date for Jacobi type weights is due to Kuijlaars and Vanlessen [2]. Let $\mu$ be absolutely continuous, and $w$ have the form

$$
w(x)=h(x) w^{(a, \beta)}(x)=h(x)(1-x)^{\alpha}(1+x)^{\beta},
$$

where $h$ is positive and analytic in $[-1,1]$. They showed that uniformly for $a, b$ in bounded subsets of $(0, \infty)$, as $n \rightarrow \infty$,

$$
\frac{1}{2 n^{2}} \tilde{K}_{n}\left(1-\frac{a}{2 n^{2}}, 1-\frac{b}{2 n^{2}}\right)=\mathbb{J}_{\alpha}(a, b)+O\left(\frac{a^{\alpha / 2} b^{\alpha / 2}}{n}\right) .
$$

Here

$$
\mathbb{J}_{\alpha}(u, v)=\frac{J_{\alpha}(\sqrt{u}) \sqrt{v} J_{\alpha}^{\prime}(\sqrt{v})-J_{\alpha}(\sqrt{v}) \sqrt{u} J_{\alpha}^{\prime}(\sqrt{u})}{2(u-v)}
$$

is the Bessel kernel of order $\alpha$, and $J_{\alpha}$ is the usual Bessel function of the first kind and order $\alpha$. Our result is: 
TheOREM 1.2. Let $\mu$ be a finite positive Borel measure on $(-1,1)$ that is regular. Assume that for some $\rho>0, \mu$ is absolutely continuous in $J=[1-\rho, 1]$, and in $J$, its absolutely continuous component has the form $w=h w^{(\alpha, \beta)}$, where $\alpha, \beta>-1$. Assume that $h(1)>0$ and $h$ is continuous at 1 . Then uniformly for $a, b$ in compact subsets of $(0, \infty)$, we have

$$
\lim _{n \rightarrow \infty} \frac{1}{2 n^{2}} \tilde{K}_{n}\left(1-\frac{a}{2 n^{2}}, 1-\frac{b}{2 n^{2}}\right)=\mathbb{J}_{\alpha}(a, b) .
$$

If $\alpha \geq 0$, we may allow compact subsets of $[0, \infty)$.

REMARKs. (a) We remind the reader that $\mu$ is regular if $w$ is positive a.e. in $(-1,1)$, or more generally if $\mu \in \mathcal{M}$.

(b) Our proof uses the fact that universality holds for the Jacobi weight $w^{(\alpha, \beta)}$.

(c) We can reformulate this in a way that allows $a, b$ to vary in a compact subset of the complex plane. To do this one shows that $n^{-2 \alpha-2} K_{n}\left(1-\frac{a}{2 n^{2}}, 1-\frac{b}{2 n^{2}}\right)$ is uniformly bounded for $n \geq 1$ and $a, b$ in compact subsets of the plane. This can be proved by bounding $\left(1-x+\frac{1}{n^{2}}\right)^{\alpha+1 / 2} K_{n}(x, x)$ in [1- $\left.\delta, 1\right]$ for some $\delta>0$, using Cauchy-Schwarz to bound $K_{n}(x, y)$, and then using the maximum principle for subharmonic functions.

This paper is organised as follows. In the next section, we establish asymptotics for Christoffel functions. In section 3, we localize, and in section 4 , we smooth, and prove the theorem. In the sequel $C, C_{1}, C_{2}, \ldots$ denote constants independent of $n, x, \theta$. The same symbol does not necessarily denote the same constant in different occurrences. We shall write $C=C(\alpha)$ or $C \neq C(\alpha)$ to respectively denote dependence on, or independence of, the parameter $\alpha$. Given measures $\mu^{*}, \mu^{\#}$, we use $K_{n}^{*}, K_{n}^{\#}$ and $p_{n}^{*}, p_{n}^{\#}$ to denote their reproducing kernels and orthonormal polynomials. Similarly superscripts $*$, \# are used to distinguish their leading coefficients and Christoffel functions, and the superscript $(\alpha, \beta)$ denotes quantities associated with the Jacobi weight $w^{(\alpha, \beta)}$.

\section{Acknowledgement}

This research was stimulated by the wonderful conference in honor of Percy Deift's 60th birthday, held at Courant Institute in June 2006.

\section{Christoffel functions}

Recall that the $n$th Christoffel function for $\mu$ is

$$
\lambda_{n}(x)=1 / K_{n}(x, x)=\min _{\operatorname{deg}(P) \leq n-1}\left(\int_{-1}^{1} P^{2} d \mu\right) / P^{2}(x) .
$$

The methods used to prove the following result are well known, but I could not find this theorem in the literature.

TheOrem 2.1. Let $\mu$ be a regular measure on $[-1,1]$. Assume that for some $\rho>0, \mu$ is absolutely continuous in $J=[1-\rho, 1]$ and in $J, w=h w^{(\alpha, \beta)}$, where $\alpha, \beta>-1$ and $h$ is bounded above and below by positive constants with

$$
\lim _{x \rightarrow 1-} h(x)=h(1)>0 .
$$

Let $A>0$. Then uniformly for $a \in[0, A]$,

$$
\lim _{n \rightarrow \infty} \lambda_{n}\left(1-\frac{a}{2 n^{2}}\right) / \lambda_{n}^{(\alpha, \beta)}\left(1-\frac{a}{2 n^{2}}\right)=h(1) .
$$


Moreover, uniformly for $n \geq n_{0}(A)$ and $a \in[0, A]$,

$$
\lambda_{n}\left(1-\frac{a}{2 n^{2}}\right) \sim \lambda_{n}^{(\alpha, \beta)}\left(1-\frac{a}{2 n^{2}}\right) \sim n^{-(2 \alpha+2) .} .
$$

The constants implicit in $\sim$ do not depend on $\rho$.

REMARKS. The notation $\sim$ means that the ratio of the two quantities is bounded above and below by positive constants independent of $n$ and $a$. Our proof actually shows that if $\left\{\varepsilon_{n}\right\}$ is any sequence of positive numbers with limit 0 ,

$$
\lambda_{n}(x) / \lambda_{n}^{(\alpha, \beta)}(x)=h(1)+o(1),
$$

uniformly for $x \in\left[1-\varepsilon_{n}, 1\right]$.

Proof: Let $\varepsilon>0$ and choose $\delta \in(0, \rho)$ such that

$$
(1+\varepsilon)^{-1} \leq \frac{h(x)}{h(1)} \leq 1+\varepsilon, \quad x \in[1-\delta, 1] .
$$

Let us define a measure $\mu^{*}$ with

$$
\mu^{*}=\mu \text { in }[-1,1-\delta)
$$

and in $I=[1-\delta, 1]$, let $\mu^{*}$ be absolutely continuous, with absolutely continuous component $w^{*}$ satisfying

$$
w^{*}=w^{(\alpha, \beta)} h(1)(1+\varepsilon) \text { in } I .
$$

Because of $(2.3), d \mu \leq d \mu^{*}$, so that if $\lambda_{n}^{*}$ is the $n$th Christoffel function for $\mu^{*}$, we have for all $x$

$$
\lambda_{n}(x) \leq \lambda_{n}^{*}(x) .
$$

We now find an upper bound for $\lambda_{n}^{*}(x)$ for $x \in[1-\delta / 2,1]$. There exists $r \in(0,1)$ such that

$$
0 \leq 1-\left(\frac{t-x}{2}\right)^{2} \leq 1-r \quad \text { for } x \in[1-\delta / 2,1] \text { and } t \in[-1,1-\delta] .
$$

In fact, we may take $r=\left(\frac{\delta}{4}\right)^{2}$. Choose $\eta \in\left(0, \frac{1}{2}\right)$ and $\sigma>1$ so close to 1 that

$$
\sigma^{1-\eta}<(1-r)^{-\eta / 4} .
$$

Let $m=m(n)=n-2[\eta n / 2]$. Fix $x \in[1-\delta / 2,1]$ and choose a polynomial $P_{m}$ of degree $\leq m-1$ such that

$$
\lambda_{m}^{(\alpha, \beta)}(x)=\int_{-1}^{1} P_{m}^{2} w^{(\alpha, \beta)} \text { and } P_{m}^{2}(x)=1 .
$$

Thus $P_{m}$ is the minimizing polynomial in the Christoffel function for the Jacobi weight $w^{(\alpha, \beta)}$ at $x$. Let

$$
S_{n}(t)=P_{m}(t)\left(1-\left(\frac{t-x}{2}\right)^{2}\right)^{[\eta n / 2]}
$$


a polynomial of degree $\leq m-1+2[\eta n / 2] \leq n-1$ with $S_{n}(x)=1$. Then using (2.4) and (2.6),

$$
\begin{aligned}
\lambda_{n}^{*}(x) & \leq \int_{-1}^{1} S_{n}^{2} d \mu^{*} \\
& \leq h(1)(1+\varepsilon) \int_{1-\delta}^{1} P_{m}^{2} w^{(\alpha, \beta)}+\left\|P_{m}\right\|_{L_{\infty}[-1,1-\delta]}^{2}(1-r)^{2[\eta n / 2]} \int_{-1}^{1-\delta} d \mu^{*} \\
& \leq h(1)(1+\varepsilon) \lambda_{m}^{(\alpha, \beta)}(x)+\left\|P_{m}\right\|_{L_{\infty}[-1,1]}^{2}(1-r)^{[2 \eta n / 2]} \int_{-1}^{1} d \mu^{*} .
\end{aligned}
$$

Now we use the key idea from $\left[4, \operatorname{Lemma} 9\right.$, p. 450]. For $m \geq m_{0}(\sigma)$, we have

$$
\begin{aligned}
\left\|P_{m}\right\|_{L_{\infty}[-1,1]}^{2} & \leq \sigma^{m} \int_{-1}^{1} P_{m}^{2} w^{(\alpha, \beta)} \\
& =\sigma^{m} \lambda_{m}^{(\alpha, \beta)}(x) .
\end{aligned}
$$

(This holds more generally for any polynomial $P$ of degree $\leq m-1$, and is a consequence of the regularity of the measure $w^{(\alpha, \beta)}$. Alternatively, we could use classic bounds for the Christoffel functions for Jacobi weight.) Then from (2.7), uniformly for $x \in[1-\delta / 2,1]$,

$$
\begin{aligned}
\lambda_{n}^{*}(x) & \leq h(1)(1+\varepsilon) \lambda_{m}^{(\alpha, \beta)}(x)\left\{1+C\left[\sigma^{1-\eta}(1-r)^{\eta / 2}\right]^{n}\right\} \\
& \leq h(1)(1+\varepsilon) \lambda_{m}^{(\alpha, \beta)}(x)\{1+o(1)\},
\end{aligned}
$$

so as $\lambda_{n} \leq \lambda_{n}^{*}$

$$
\begin{aligned}
& \sup _{x \in[1-\delta / 2,1]} \lambda_{n}(x) / \lambda_{n}^{(\alpha, \beta)}(x) \\
& \leq h(1)(1+\varepsilon)\{1+o(1)\} \sup _{x \in[1-\delta / 2,1]} \lambda_{m}^{(\alpha, \beta)}(x) / \lambda_{n}^{(\alpha, \beta)}(x) .
\end{aligned}
$$

Now for large enough $n$, and some $C$ independent of $\delta, \eta, m, n$,

$$
\sup _{x \in[1-\delta / 2,1]} \lambda_{m}^{(\alpha, \beta)}(x) / \lambda_{n}^{(\alpha, \beta)}(x) \leq 1+C \eta .
$$

Indeed if $\left\{p_{k}^{(\alpha, \beta)}\right\}$ denote the orthonormal Jacobi polynomials for $w^{(\alpha, \beta)}$, they admit the bound [5, p. 170]

$$
\left|p_{k}^{(\alpha, \beta)}(x)\right| \leq C\left(1-x+\frac{1}{k^{2}}\right)^{-\alpha / 2-1 / 4}, \quad x \in[0,1] .
$$

Then

$$
\begin{aligned}
0 & \leq 1-\frac{\lambda_{n}^{(\alpha, \beta)}(x)}{\lambda_{m}^{(\alpha, \beta)}(x)}=\lambda_{n}^{(\alpha, \beta)}(x) \sum_{k=m}^{n-1}\left(p_{k}^{(\alpha, \beta)}(x)\right)^{2} \\
& \leq C \lambda_{n}^{(\alpha, \beta)}(x)(n-m) \max _{\frac{n}{2} \leq k \leq n}\left(1-x+\frac{1}{k^{2}}\right)^{-\alpha-1 / 2} \\
& \leq C \eta n \lambda_{n}^{(\alpha, \beta)}(x)\left(1-x+\frac{1}{n^{2}}\right)^{-\alpha-1 / 2} \\
& \leq C \eta,
\end{aligned}
$$

by classical bounds for Christoffel functions [5, p. 108, Lemma 5]. So we have (2.9). 
Now let $a \in[0, A]$. We see that for $n \geq n_{0}(A)$, we have $1-a /\left(2 n^{2}\right) \in$ $[1-\delta / 2,1]$, and hence $(2.8)$ gives

$$
\limsup _{n \rightarrow \infty}\left(\sup _{a \in[0, A]} \lambda_{n}\left(1-\frac{a}{2 n^{2}}\right) / \lambda_{n}^{(\alpha, \beta)}\left(1-\frac{a}{2 n^{2}}\right)\right) \leq h(1)(1+\varepsilon)(1+C \eta) .
$$

As the left-hand side is independent of the parameters $\varepsilon, \eta$, we deduce that

$$
\limsup _{n \rightarrow \infty}\left(\sup _{a \in[0, A]} \lambda_{n}\left(1-\frac{a}{2 n^{2}}\right) / \lambda_{n}^{(\alpha, \beta)}\left(1-\frac{a}{2 n^{2}}\right)\right) \leq h(1) .
$$

In a similar way, we can establish the converse bound

$$
\limsup _{n \rightarrow \infty}\left(\sup _{a \in[0, A]} \lambda_{n}^{(\alpha, \beta)}\left(1-\frac{a}{2 n^{2}}\right) / \lambda_{n}\left(1-\frac{a}{2 n^{2}}\right)\right) \leq h(1)^{-1} .
$$

Indeed with $m, x$ and $\eta$ as above, let us choose a polynomial $P$ of degree $\leq m-1$ such that

$$
\lambda_{m}(x)=\int_{-1}^{1} P_{m}^{2}(t) d \mu(t) \text { and } P_{m}^{2}(x)=1 .
$$

Then with $S_{n}$ as above, and proceeding as above,

$$
\begin{aligned}
& \lambda_{n}^{(\alpha, \beta)}(x) \leq \int_{-1}^{1} S_{n}^{2} w^{(\alpha, \beta)} \\
& \quad \leq\left[h(1)^{-1}(1+\varepsilon)\right] \int_{1-\delta}^{1} P_{m}^{2} d \mu+\left\|P_{m}\right\|_{L_{\infty}[-1,1-\delta]}^{2}(1-r)^{2[\eta n / 2]} \int_{-1}^{1-\delta} w^{(\alpha, \beta)} \\
& \quad \leq\left[h(1)^{-1}(1+\varepsilon)\right] \lambda_{m}(x)\left\{1+C\left[\sigma^{1-\eta}(1-r)^{\eta / 2}\right]^{n}\right\}
\end{aligned}
$$

and so as above,

$$
\begin{aligned}
& \sup _{x \in[1-\delta / 2,1]} \lambda_{m}^{(\alpha, \beta)}(x) / \lambda_{m}(x) \\
\leq & {\left[h(1)^{-1}(1+\varepsilon)(1+o(1))\right] \sup _{x \in[1-\delta / 2,1]} \lambda_{m}^{(\alpha, \beta)}(x) / \lambda_{n}^{(\alpha, \beta)}(x) } \\
\leq & {\left[h(1)^{-1}(1+\varepsilon)\right]\{1+o(1)\}(1+C \eta) . }
\end{aligned}
$$

Then (2.11) follows after a scale change $m \rightarrow n$ and using monotonicity of $\lambda_{n}$ in $n$. Together (2.10) and (2.11) give the result.

\section{Localization}

TheOrem 3.1. Assume that $\mu, \mu^{*}$ are regular measures on $[-1,1]$. Assume that

$$
d \mu=d \mu^{*}=\left(h w^{(\alpha, \beta)}\right)(t) d t \text { in } J=[1-\rho, 1],
$$

where $h$ satisfies the hypothesis of Theorem 1.2. Let $A>0$. Then as $n \rightarrow \infty$,

$$
\sup _{a, b \in[0, A]}\left|\left(K_{n}-K_{n}^{*}\right)\left(1-\frac{a}{2 n^{2}}, 1-\frac{b}{2 n^{2}}\right)\right| / n^{2 \alpha+2}=o(1) .
$$


Proof: We initially assume that

$$
d \mu \leq d \mu^{*} \text { in }(-1,1) .
$$

The idea is to estimate the $L_{2}$ norm of $K_{n}(x, t)-K_{n}^{*}(x, t)$ over $[-1,1]$, and then to use Christoffel function estimates. Now

$$
\begin{aligned}
& \int_{-1}^{1}\left(K_{n}(x, t)-K_{n}^{*}(x, t)\right)^{2} d \mu(t) \\
& =\int_{-1}^{1} K_{n}^{2}(x, t) d \mu(t)-2 \int_{-1}^{1} K_{n}(x, t) K_{n}^{*}(x, t) d \mu(t)+\int_{-1}^{1} K_{n}^{* 2}(x, t) d \mu(t) \\
& =K_{n}(x, x)-2 K_{n}^{*}(x, x)+\int_{-1}^{1} K_{n}^{* 2}(x, t) d \mu(t),
\end{aligned}
$$

by the reproducing kernel property. As $d \mu \leq d \mu^{*}$, we also have

$$
\int_{-1}^{1} K_{n}^{* 2}(x, t) d \mu(t) \leq \int_{-1}^{1} K_{n}^{* 2}(x, t) d \mu^{*}(t)=K_{n}^{*}(x, x) .
$$

So

$$
\int_{-1}^{1}\left(K_{n}(x, t)-K_{n}^{*}(x, t)\right)^{2} d \mu(t) \leq K_{n}(x, x)-K_{n}^{*}(x, x) .
$$

Next for any polynomial $P$ of degree $\leq n-1$, we have the Christoffel function estimate

$$
|P(y)| \leq K_{n}(y, y)^{1 / 2}\left(\int_{-1}^{1} P^{2} d \mu\right)^{1 / 2} .
$$

Applying this to $P(t)=K_{n}(x, t)-K_{n}^{*}(x, t)$ and using (3.3) gives

$$
\left|K_{n}(x, y)-K_{n}^{*}(x, y)\right| \leq K_{n}(y, y)^{1 / 2}\left[K_{n}(x, x)-K_{n}^{*}(x, x)\right]^{1 / 2}
$$

so

$$
\frac{\left|K_{n}(x, y)-K_{n}^{*}(x, y)\right|}{K_{n}(x, x)} \leq\left(\frac{K_{n}(y, y)}{K_{n}(x, x)}\right)^{1 / 2}\left[1-\frac{K_{n}^{*}(x, x)}{K_{n}(x, x)}\right]^{1 / 2} .
$$

Now we set $x=1-\frac{a}{2 n^{2}}$ and $y=1-\frac{b}{2 n^{2}}$, where $a, b \in[0, A]$. By Theorem 2.1, uniformly for such $x, \frac{K_{n}^{*}(x, x)}{K_{n}(x, x)}=1+o(1)$, for they both have the same asymptotics as for the Jacobi weight. Moreover, uniformly for $a, b \in[0, A]$,

$$
K_{n}\left(1-\frac{b}{2 n^{2}}, 1-\frac{b}{2 n^{2}}\right) \sim K_{n}\left(1-\frac{a}{2 n^{2}}, 1-\frac{a}{2 n^{2}}\right) \sim n^{2 \alpha+2},
$$

so

$$
\sup _{a, b \in[0, A]}\left|\left(K_{n}-K_{n}^{*}\right)\left(1-\frac{a}{2 n^{2}}, 1-\frac{b}{2 n^{2}}\right)\right| / n^{2 \alpha+2}=o(1) .
$$

Now we drop the extra hypothesis (3.2). Define a measure $\nu$ by $\nu=\mu=\mu^{*}$ in $J$ and

$$
d \nu(x)=\max \left\{1, w, w^{*}\right\} d x+d \mu_{s}+d \mu_{s}^{*}, \text { in }[-1,1] \backslash J
$$

where $w, w^{*}$ and $\mu_{s}, \mu_{s}^{*}$ are respectively the absolutely continuous and singular components of $\mu, \mu^{*}$. Then $d \mu \leq d \nu$ and $d \mu^{*} \leq d \nu$, and $\nu$ is regular as its absolutely continuous component is positive in $(-1,1)$, and hence lies in the even smaller class $\mathcal{M}$. The case above shows that the reproducing kernels for $\mu$ and $\mu^{*}$ have the same asymptotics as that for $\nu$, in the sense of (3.1), and hence the same asymptotics as each other. 


\section{Smoothing}

In this section, we approximate $\mu$ of Theorem 1.2 by a Jacobi measure $\mu^{\#}$ and then prove Theorem 1.2. Our smoothing result is:

Theorem 4.1. Let $\mu$ be as in Theorem 1.2. Let $\varepsilon \in\left(0, \frac{1}{2}\right)$ and choose $\delta>0$ such that (2.3) holds. Let

$$
w^{\#}=h(1) w^{(\alpha, \beta)} \text { in }(-1,1) .
$$

Let $A>0$. Then there exists $C$ and $n_{0}$ such that for $n \geq n_{0}$,

$$
\sup _{a, b \in[0, A]}\left|\left(K_{n}-K_{n}^{\#}\right)\left(1-\frac{a}{2 n^{2}}, 1-\frac{b}{2 n^{2}}\right)\right| / n^{2 \alpha+2} \leq C \varepsilon^{1 / 2},
$$

where $C$ is independent of $\varepsilon, n$.

Proof: We note that because of our localization result Theorem 3.1, we may replace $w$ by $w^{*}$, where

$$
w^{*}=w=w^{(\alpha, \beta)} h \text { in } I=[1-\delta, 1]
$$

and

$$
w^{*}=w^{(\alpha, \beta)} h(1) \text { in }[-1,1] \backslash I,
$$

without affecting the asymptotics for $K_{n}\left(1-\frac{a}{2 n^{2}}, 1-\frac{b}{2 n^{2}}\right)$. (Note that $\varepsilon$ and $\delta$ play no role in Theorem 3.1.) So in the sequel, we assume that $w=w^{(\alpha, \beta)} h(1)=w^{\#}$ in $[-1,1] \backslash I$, while keeping $w$ the same in $I$. Observe that (2.3) implies that

$$
(1+\varepsilon)^{-1} \leq \frac{w}{w^{\#}} \leq 1+\varepsilon, \text { in }[-1,1] .
$$

Then, much as in the previous section,

$$
\begin{aligned}
& \int_{-1}^{1}\left(K_{n}(x, t)-K_{n}^{\#}(x, t)\right)^{2} w^{\#}(t) d t \\
& =\int_{-1}^{1} K_{n}^{2}(x, t) w^{\#}(t) d t-2 \int_{-1}^{1} K_{n}(x, t) K_{n}^{\#}(x, t) w^{\#}(t) d t \\
& \quad+\int_{-1}^{1} K_{n}^{\# 2}(x, t) w^{\#}(t) d t \\
& =\int_{-1}^{1} K_{n}^{2}(x, t) w(t) d t+\int_{I} K_{n}^{2}(x, t)\left(w^{\#}-w\right)(t) d t-2 K_{n}(x, x)+K_{n}^{\#}(x, x) \\
& =K_{n}^{\#}(x, x)-K_{n}(x, x)+\int_{I} K_{n}^{2}(x, t)\left(w^{\#}-w\right)(t) d t,
\end{aligned}
$$

recall that $w=w^{\#}$ in $[-1,1] \backslash I$. By $(4.3)$,

$$
\int_{I} K_{n}^{2}(x, t)\left(w^{\#}-w\right)(t) d t \leq \varepsilon \int_{I} K_{n}^{2}(x, t) w(t) d t \leq \varepsilon K_{n}(x, x) .
$$

So

$$
\int_{-1}^{1}\left(K_{n}(x, t)-K_{n}^{\#}(x, t)\right)^{2} w^{\#}(t) d t \leq K_{n}^{\#}(x, x)-(1-\varepsilon) K_{n}(x, x) .
$$

Applying an obvious analogue of (3.4) to $P(t)=K_{n}(x, t)-K_{n}^{\#}(x, t)$ and using (4.4) gives for $y \in[-1,1]$,

$$
\left|K_{n}(x, y)-K_{n}^{\#}(x, y)\right| \leq K_{n}^{\#}(y, y)^{1 / 2}\left[K_{n}^{\#}(x, x)-(1-\varepsilon) K_{n}(x, x)\right]^{1 / 2}
$$


SO

$$
\frac{\left|K_{n}(x, y)-K_{n}^{\#}(x, y)\right|}{K_{n}^{\#}(x, x)} \leq\left(\frac{K_{n}^{\#}(y, y)}{K_{n}^{\#}(x, x)}\right)^{1 / 2}\left[1-(1-\varepsilon) \frac{K_{n}(x, x)}{K_{n}^{\#}(x, x)}\right]^{1 / 2} .
$$

In view of $(4.3)$, we also have

$$
\frac{K_{n}(x, x)}{K_{n}^{\#}(x, x)}=\frac{\lambda_{n}^{\#}(x)}{\lambda_{n}(x)} \geq \frac{1}{1+\varepsilon},
$$

so for all $y \in[-1,1]$,

$$
\begin{aligned}
\frac{\left|K_{n}(x, y)-K_{n}^{\#}(x, y)\right|}{K_{n}^{\#}(x, x)} & \leq\left(\frac{K_{n}^{\#}(y, y)}{K_{n}^{\#}(x, x)}\right)^{1 / 2}\left[1-\frac{1-\varepsilon}{1+\varepsilon}\right]^{1 / 2} \\
& \leq \sqrt{2 \varepsilon}\left(\frac{K_{n}^{\#}(y, y)}{K_{n}^{\#}(x, x)}\right)^{1 / 2} .
\end{aligned}
$$

Now we set $x=1-\frac{a}{2 n^{2}}$ and $y=1-\frac{b}{2 n^{2}}$, where $a, b \in[0, A]$. By Theorem 2.1, uniformly for $a, b \in[0, A]$,

$$
K_{n}^{\#}\left(1-\frac{b}{2 n^{2}}, 1-\frac{b}{2 n^{2}}\right) \sim K_{n}^{\#}\left(1-\frac{a}{2 n^{2}}, 1-\frac{a}{2 n^{2}}\right) \sim n^{2 \alpha+2},
$$

and also the constants implicit in $\sim$ are independent of $\varepsilon$ (this is crucial!). Thus for some $C$ and $n_{0}$ depending only on $A$, we have for $n \geq n_{0}$,

$$
\sup _{a, b \in[0, A]}\left|\left(K_{n}-K_{n}^{\#}\right)\left(1-\frac{a}{2 n^{2}}, 1-\frac{b}{2 n^{2}}\right)\right| / n^{2 \alpha+2} \leq C \sqrt{\varepsilon} .
$$

Proof of Theorem 1.2: Let $\varepsilon_{1}>0$. We can choose $\varepsilon>0$ so small that the right-hand side of (4.2) is less than $\varepsilon_{1}$. (Recall that $C$ there is independent of $\varepsilon$.) Hence for $n \geq n_{0}\left(A, \varepsilon_{1}\right)$,

$$
\sup _{a, b \in[0, A]}\left|\left(K_{n}-K_{n}^{\#}\right)\left(1-\frac{a}{2 n^{2}}, 1-\frac{b}{2 n^{2}}\right)\right| / n^{2 \alpha+2} \leq \varepsilon_{1} .
$$

It follows that

$$
\lim _{n \rightarrow \infty}\left(\sup _{a, b \in[0, A]}\left|\left(K_{n}-K_{n}^{\#}\right)\left(1-\frac{a}{2 n^{2}}, 1-\frac{b}{2 n^{2}}\right)\right| / n^{2 \alpha+2}\right)=0 .
$$

Next, uniformly for $a \in\left[A_{1}, A_{2}\right]$, where $0<A_{1}<A_{2}<\infty$, we see that

$$
w\left(1-\frac{a}{2 n^{2}}\right)=(1+o(1)) h(1) 2^{\beta}\left(\frac{a}{2 n^{2}}\right)^{\alpha}=w^{\#}\left(1-\frac{a}{2 n^{2}}\right)(1+o(1)),
$$

with a similar relation when we replace $a$ by $b$. Hence uniformly for $a, b \in\left[A_{1}, A_{2}\right]$,

$$
\begin{aligned}
& \frac{1}{2 n^{2}} \tilde{K}_{n}\left(1-\frac{a}{2 n^{2}}, 1-\frac{b}{2 n^{2}}\right) \\
& =\frac{1}{2 n^{2}} \tilde{K}_{n}^{\#}\left(1-\frac{a}{2 n^{2}}, 1-\frac{b}{2 n^{2}}\right)(1+o(1))+o(1) \\
& =\mathbb{J}_{\alpha}(a, b)+o(1),
\end{aligned}
$$

by the universality limit (1.2) for the scaled Jacobi weight $w^{\#}=h(1) w^{(\alpha, \beta)}$. For this, see for example [2]. When $\alpha \geq 0$, we can allow instead $a \in[0, A]$. 


\section{References}

[1] P. Deift, Orthogonal Polynomials and Random Matrices: A Riemann-Hilbert Approach, Courant Institute Lecture Notes, Vol. 3, New York University Pres, New York, 1999.

[2] A.B. Kuijlaars and M. Vanlessen, Universality for Eigenvalue Correlations from the Modified Jacobi Unitary Ensemble, International Maths. Research Notices, 30(2002), 1575-1600.

[3] D.S. Lubinsky, A New Approach to Universality Limits involving Orthogonal Polynomials, to appear in Annals of Mathematics.

[4] A. Mate, P. Nevai, V. Totik, Szego's Extremum Problem on the Unit Circle, Annals of Math., 134(1991), 433-453.

[5] P. Nevai, Orthogonal Polynomials, Memoirs of the AMS no. 213 (1979).

[6] B. Simon, Orthogonal Polynomials on the Unit Circle, Parts 1 and 2, American Mathematical Society, Providence, 2005.

[7] H. Stahl and V. Totik, General Orthogonal Polynomials, Cambridge University Press, Cambridge, 1992.

School of Mathematics, Georgia Institute of Technology, Atlanta, GA 30332-0160 USA

E-mail address: lubinsky@math.gatech.edu 